\title{
BMJ Open Sino Longitudinal Study on Cognitive Decline (SILCODE): protocol for a Chinese longitudinal observational study to develop risk prediction models of conversion to mild cognitive impairment in individuals with subjective cognitive decline
}

\author{
Xuanyu Li, ${ }^{1}$ Xiaoni Wang, ${ }^{\oplus 1}$ Li Su, ${ }^{2,3}$ Xiaochen Hu, ${ }^{4}$ Ying Han ${ }^{\odot 1,5,6,7}$
}

To cite: Li X, Wang X, Su L, et al. Sino Longitudinal Study on Cognitive Decline (SILCODE): protocol for a Chinese longitudinal observational study to develop risk prediction models of conversion to mild cognitive impairment in individuals with subjective cognitive decline. BMJ Open 2019;9:e028188. doi:10.1136/ bmjopen-2018-028188

- Prepublication history for this paper is available online. To view these files, please visit the journal online (http://dx.doi. org/10.1136/bmjopen-2018028188)

$\mathrm{XL}$ and $\mathrm{XW}$ contributed equally.

Received 26 November 2018 Revised 15 May 2019 Accepted 25 June 2019

Check for updates

(c) Author(s) (or their employer(s)) 2019. Re-use permitted under CC BY-NC. No commercial re-use. See rights and permissions. Published by BMJ.

For numbered affiliations see end of article.

Correspondence to

Dr Ying Han;

hanying@xwh.ccmu.edu.cn

\section{ABSTRACT}

Introduction Understanding the biological mechanism of subjective cognitive decline (SCD) in preclinical Alzheimer's disease (AD) and identifying those who will soon convert to mild cognitive impairment (MCl) are critical for developing appropriate strategies for early diagnosis and intervention of AD. We present the study protocol of the Sino Longitudinal Study on Cognitive Decline (SILCODE), a longitudinal observational study focusing on SCD in the context of AD. Methods and analysis Within SILCODE, approximately 800 subjects with SCD who are between 50 and 79 years old will be recruited through standardised public advertisements or memory clinics. They will undergo extensive assessment, including clinical and neuropsychological assessments, blood sample collection for plasma beta-amyloid and ApoE genotype, urine samples collection for AD7c-NTP, and multimodal MRI scans (structural MRI, diffusion tensor imaging, resting-state functional MRI and optional task-based functional MRI) as well as optional glucose metabolism and amyloid positron emission tomography. Subjects will be contacted by telephone every 3 months and interviewed, on average, every 15 months for 5 years. The study endpoint is the development of mild cognitive impairment or dementia. Jak \& Bondi's actuarial neuropsychological method will be used for diagnosis of $\mathrm{MCl}$. The least absolute shrinkage and selection operator logistic regression model followed by the sub-distribution hazard function model with death as a competing risk will be constructed to establish risk prediction models. Ethics and dissemination The ethics committee of the Xuanwu Hospital of Capital Medical University has approved this study protocol (ID: [2017]046). The results will be published in peer-reviewed journals and presented at national and international scientific conferences. Trial registration number NCT03370744; Pre-results.

\section{INTRODUCTION}

Alzheimer's disease (AD) is the most common neurodegenerative disease and one
Strengths and limitations of this study

- A strength of this study is the use of multiple markers (clinical, blood, urine and imaging markers) to establish models predicting conversion from subjective cognitive decline (SCD) to mild cognitive impairment, which will provide references for clinical applications of SCD.

- This longitudinal study could enrich the understanding of SCD by examining a large-scale Chinese population because cultural factors have an impact on SCD.

- The relatively short follow-up duration (5 years) of this study allows longitudinal data to be analysed only in an exploratory way.

- The study results will be limited by the research environment in that individuals will be recruited from memory clinics and volunteer samples, without community-based or population-based samples.

of the greatest healthcare challenges of the 21 st century. ${ }^{1}$ It has been revealed that pathophysiology starts many years before the onset of clinical symptoms. ${ }^{2}$ Several recent clinical trials of $\beta$-amyloid $(A \beta)$-lowering therapies in the mild or moderate dementia stage and even mild cognitive impairment stage have failed, ${ }^{34}$ further encouraging researchers to shift their focus to the preclinical stage of AD as a target for new treatments. ${ }^{5}$

Subjective cognitive decline (SCD) refers to self-perceived cognitive decline relative to a previously normal status, without impaired performance on standardised neuropsychological tests. ${ }^{67}$ Evidence from recent studies suggests that SCD may be one of the earliest symptomatic manifestations of AD ${ }^{68}$ Previous 
studies have found that individuals with SCD have a higher conversion rate and shorter conversion time to mild cognitive impairment (MCI) and dementia than normal controls. ${ }^{9-12}$ SCD may be an early trigger for helpseeking, ${ }^{13}$ and we have observed that the number of individuals with SCD presenting to the healthcare system (eg, memory clinics) is increasing in our clinical practice as awareness of $\mathrm{AD}$ dementia increases in China. Individuals with SCD are ideal for ultra-early diagnosis and intervention trials.

However, the heterogeneous nature of SCD limits its value in clinical applications. Research to date has focused on factors for predicting the risk of MCI/AD progression in SCD. ${ }^{14-17}$ Though some longitudinal and observational studies of SCD are ongoing, ${ }^{18-20}$ there are few data available from China or anywhere else in Asia. However, SCD is subject to influence by various cultural factors including factors that relate to language, social stigmatisation of cognitive problems, styles of responding on self-reported measures (eg, extremity of answers), tolerance of slowly progressive cognitive decline in ageing and others. ${ }^{21}$ The association between objective cognitive performance and SCD is different across races. ${ }^{22}$ Our group reported that the prevalence of SCD was $14.4 \%$ in the Shunyi District of Beijing, China, ${ }^{23}$ which is different from the prevalence of SCD reported in Western countries. ${ }^{24}{ }^{25}$ Thus, there is a need for additional findings from longitudinal studies based on large-scale Chinese populations.

The Alliance for Preclinical AD in China, belonging to the National Clinical Research Center for Geriatric Disorders, is a national multicentre research platform that is dedicated to the development of clinical, neuroimaging, genetic and biochemical biomarkers for the early detection and tracking of $\mathrm{AD}$. Its sites include more than 180 member hospitals/research institutes across China. The alliance is led by Professor Han and focuses on the early detection of SCD during preclinical $\mathrm{AD}$; it also develops and provides standard operating procedures and quality control for harmonised data and material acquisition and storage across all sites.

In this protocol, we introduce in detail the design of the Sino Longitudinal Study on Cognitive Decline (SILCODE) study. This is an ongoing longitudinal observational study based on the multicentre research platform of the Alliance for Preclinical AD in China and focusing on SCD in the context of AD. The main aim of SILCODE is to estimate cognitive decline and establish statistical prediction models for the outcomes of SCD in elderly Chinese people.

\section{METHODS \\ Overall study design}

This observational longitudinal study will take place in China and will recruit people with SCD. During the baseline period, all eligible subjects with SCD will be invited to receive clinical and neuropsychological examinations, blood tests, urine tests and multimodal MRI (including structural MRI (sMRI), diffusion tensor imaging (DTI) and resting-state functional MRI (rs-fMRI)) sequentially as shown in figure 1. Glucose metabolism and amyloid positron emission tomography (PET) and task-based fMRI (t-fMRI) will be performed as well if the subject agrees to them. A semi-structured interview ${ }^{18}$ as well as the SCD questionnaire ${ }^{26}$ and the Measurement of Everyday Cognition (ECog) ${ }^{27}$ will be used to assess SCD. The maximum interval between neuropsychological tests and other data collection will be within 3 months. Subjects will be contacted by telephone every 3 months and will be interviewed, on average, every 15 months for 5 years (four time points). The Montreal Cognitive Assessment-Basic (MoCA-B) ${ }^{28}$ subtests (language, delayed

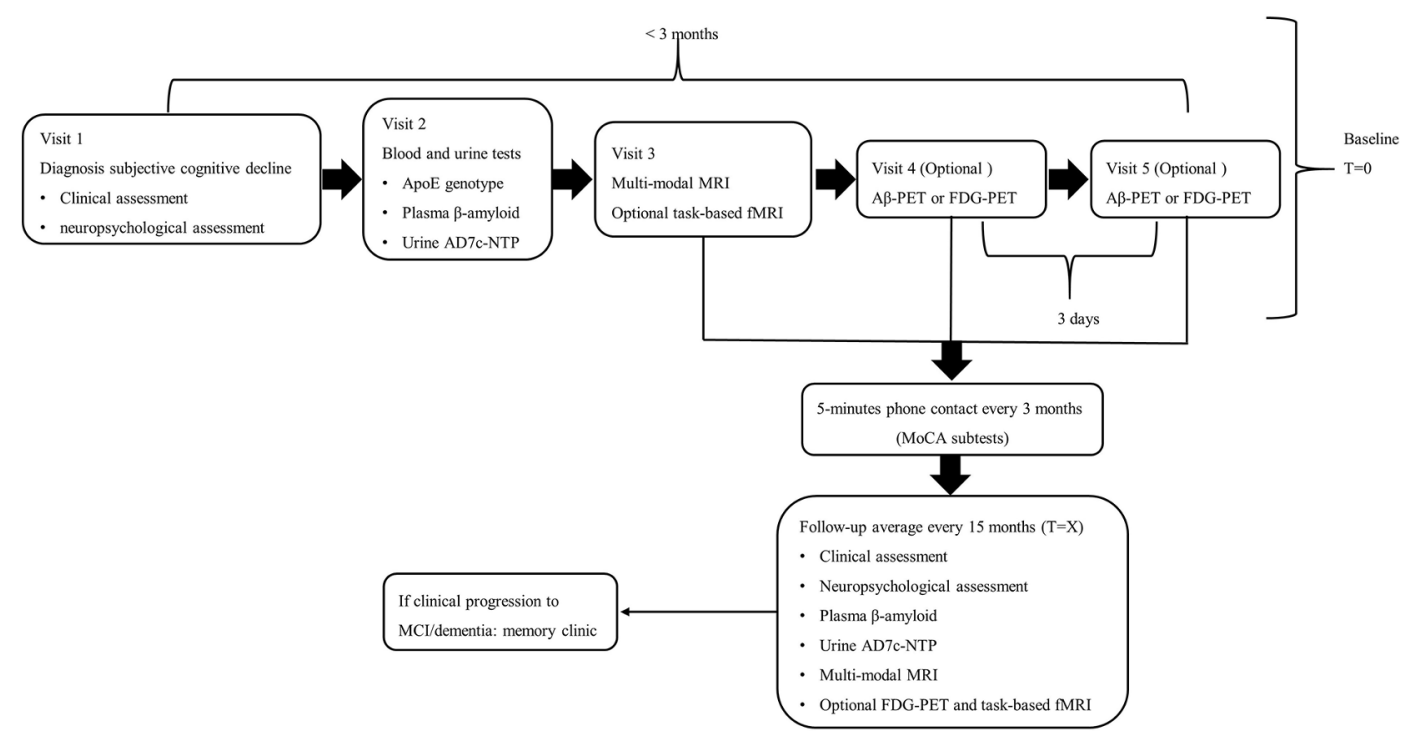

Figure 1 Flow chart of SILCODE. A $\beta$-PET, $\beta$-amyloid positron emission tomography; fMRI, functional MRI; FDG-PET, fluorodeoxyglucose positron emission tomography; MoCA: Montreal Cognitive Assessment-basic; SCD, subjective cognitive decline. 
recall and orientation) will be administered during the call. Participants expressing significant cognitive decline on the telephone will be interviewed ahead of time for the next follow-up. During the follow-up visits with interviews, evaluations including clinical and neuropsychological examinations, blood tests, urine tests and multimodal MRI as well as optional glucose metabolism PET and t-fMRI will be conducted, on average, every 15 months. At each follow-up visit, diagnoses will be re-evaluated under the supervision of two neurologists. Figure 1 provides the overall study outline.

\section{Patient and public involvement}

Patients and the public will not be involved in the development of the research question or the design of the study. Patients will not be involved in the recruitment of participants or the conduct of the study. The general results will be disseminated to participants through public education activities.

\section{Sample size calculation}

The formula proposed by Hsieh and Lavori ${ }^{29}$ was used to estimate the sample size in our study with the software PASS V.15.0.5. According to a previous meta-analysis, ${ }^{9}$ the cumulative conversion proportion was $24.4 \%$ over a mean follow-up period of 4.1 years. We assumed that the power $(1-\beta)$ was $80 \%$, a was 0.05 and the rate of loss to follow-up was $20 \%$. Due to the lack of statistical data on SCD, we calculated the sample size with data reported by Licher et $a l^{30}$ and Pinto et $a \hat{l}^{31} \quad\left(\mathrm{HR}=1.03, \mathrm{SD}=8.2, \mathrm{R}^{2}=0.127\right)$ from cognitively normal elderly subjects for the association between age and cognitive decline. This calculation rendered a total sample size of 762 . Considering the need for internal validation, we will recruit at least 800 subjects.

\section{Recruitment of participants}

Participants will be consecutively recruited through standardised public advertisements and through referrals from general physicians, memory clinics, or informants. Residents who meet the inclusion criteria will be recruited. Written informed consent will be acquired from each subject before enrolment.

\section{Diagnostic criteria for SCD, $\mathrm{MCl}$ and dementia}

SCD is defined by the research criteria for pre-MCI (SCD) proposed by Jessen et al in $2014^{6}$ : (1) Self-experienced persistent decline in cognitive capacity in comparison with a previously normal status and unrelated to an acute event; (2) Normal age-adjusted, gender-adjusted and education-adjusted performance on standardised cognitive tests.

MCI is defined by an actuarial neuropsychological method proposed by Jak and Bondi. ${ }^{32}$ Participants are considered to have MCI if they meet any one of the following three criteria and fail to meet the criteria for dementia: (1) having impaired scores (defined as >1 SD below the age-corrected normative means) on both measures in at least one cognitive domain (memory, language, or speed/executive function); (2) having impaired scores in each of the three cognitive domains sampled (memory, language, or speed/executive function); (3) the Functional Activities Questionnaire (FAQ) $\geq 9$. The normative means in our study are from Guo and his team in a Chinese population. ${ }^{33-36}$ Measures and normative means are shown in table 1 .

The diagnosis of $\mathrm{AD}$ dementia is based on the Diagnostic and Statistical Manual of Mental Disorders, fifth edition, and the diagnostic guidelines for dementia due to $\mathrm{AD}$ delivered by the National Institute on AgeingAlzheimer's Association workgroups,${ }^{37}$ and a total CDR score $\geq 1$.

\section{Inclusion/exclusion criteria}

The inclusion criteria are as follows: (1) 50-79 years old, right-handed and Mandarin-speaking subjects; (2) presence of self-perceived continuous cognitive decline compared with previous normal status and unrelated to an acute event; (3) concerns (worries) associated with memory complaint; and (4) failure to meet the criteria for MCI or dementia.

The exclusion criteria are as follows: (1) History of stroke. (2) Current major psychiatric diagnoses such as severe depression and anxiety. When mild or moderate symptoms of psychiatric disorders are suspected, patients will not be excluded. ${ }^{38}$ They will be evaluated by a psychiatrist to determine whether the psychiatric diagnoses

Table 1 Cognitive domains, neuropsychological tests and normative means

\begin{tabular}{lll} 
Cognitive domains & Neuropsychological tests & Normative means \\
\hline Memory & Auditory Verbal Learning Test-long delayed & 50-59years old: 5; 60-69years old: 4; \\
& memory & $70-79$ years old: 3
\end{tabular}


are the cause of SCD. (3) Other neurological conditions that could cause cognitive decline (eg, brain tumours, Parkinson's disease, encephalitis, or epilepsy) rather than $\mathrm{AD}$ spectrum disorders. (4) Other diseases that could cause cognitive decline (eg, thyroid dysfunction, severe anaemia, syphilis, or HIV). (5) History of psychosis or congenital mental developmental delay. (6) Cognitive decline caused by traumatic brain injury. (7) Inability to complete the study protocol or presence of contraindications for MRI.

\section{Clinical progression}

At each follow-up visit, diagnoses will be re-evaluated under the supervision of two neurologists. The main outcome measure of clinical progression in our study is conversion to MCI or $\mathrm{AD}$ dementia. The time to event is the interval between the baseline neuropsychological tests and the date at which the MCI is first diagnosed. For those who bypass the diagnosis of MCI during follow-up, the time between the baseline neuropsychological tests and first diagnosis of dementia is defined as the time to event in our study.

\section{Assessments of subjective cognitive functioning}

A semi-structured interview used by the Multicenter German Center for Neurodegenerative Diseases (DZNE)-Longitudinal Cognitive Impairment and Dementia Study (DELCODE) study will be employed in this project to evaluate the details of SCD. ${ }^{18}$ It includes information about the onset time, concerns, comparison with others, and the history of visiting a physician not only for the memory domain but also for language, attention and executive control. We will also require informant reports in the evaluation of the self-reported information as suggested by the Subjective Cognitive Decline Initiative Working Group. $^{739}$

For quantitative assessment of the severity of SCD, we will use a newly developed SCD questionnaire including nine reliable SCD items (box 1), which are characterised by different domains, such as global memory functioning

Box 1 Items included in subjective cognitive decline questionnaire ${ }^{26}$

Items

Do you think you have problems with your memory?

- Do you have difficulty remembering a conversation from a few days ago?

Do you have complaints about your memory in the last 2 years?

How often is the following a problem for you: Personal dates?

- How often is the following a problem for you: Phone numbers you use frequently?

- On a whole, do you think that you have problems remembering things that you want to do or say?

- How often is the following a problem for you: Going to the store and forgetting what you wanted to buy?

Do you think that your memory is worse than 5 years ago?

Do you feel you are forgetting where things were placed? and daily activities ability. ${ }^{23}{ }^{26}$ In addition, the 12-item $\mathrm{ECog}^{27}$ will also be applied to all participants to measure SCD severity. These questionnaires will not be inclusion criteria and will be considered only for statistical analysis.

\section{Clinical, risk factor and neuropsychological assessments}

The clinical assessments include a structured medical history, physical examination, routine laboratory tests (including blood tests, biochemical tests, thyroid function tests, vitamin B12 status, folic acid status, Treponema pallidum-specific antibodies and HIV antibodies) and MRI (T1 and FLAIR) for medial temporal atrophy scale and Fazekas score.

For risk factors of $\mathrm{AD}$, all subjects and their informants will provide the following information: family history, vascular risk factors, occupation/retirement, socioeconomic status, cigarettes and alcohol use, and nutrition style. Additionally, the Pittsburgh sleep quality index, ${ }^{40}$ Rapid Eye Movement Sleep Behaviour Disorder Screening Questionnaire ${ }^{41}$ and the Epworth Sleepiness Scale ${ }^{42}$ will be used for sleep quality assessment.

The neuropsychological tests are selected for comparability with other similar ongoing studies (eg, DELCODE). ${ }^{18}$ To avoid deviations in the evaluation, the neuropsychologists performing the cognitive tests will be trained according to standard guidelines. The kappa coefficient (Fleiss' kappa) will be used to measure the assessment agreement among the neuropsychologists. Neuropsychological results will be double-checked. The neuropsychological battery (table 1) measures different cognitive domains including episodic memory (Auditory Verbal Learning Test-HuaShan version), ${ }^{33}$ language (Animal Fluency Test, ${ }^{35}$ 30-item Boston Naming Test ${ }^{34}$ and speed/executive function (Shape Trail Test Parts A and $\mathrm{B} .{ }^{36}$ In addition, the SILCODE study will implement tests for global cognition, daily life ability and neuropsychiatric assessment, including MoCA-B, ${ }^{28}$ the memory and executive screening (MES) ${ }^{43}$ the CDR, FAQ, the 15-item short form of the Geriatric Depression Scale, the Hamilton Anxiety Scale, the Hamilton Depression Scale and the Neuropsychiatric Inventory.

\section{Blood tests}

\section{ApoE genotype}

Single nucleotide polymorphisms (SNPs) rs7412 and

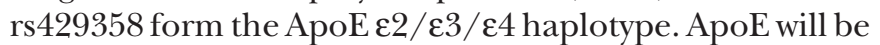
genotyped using the standard Sanger sequencing method (Sangon, Shanghai, China) using the following primers: 5'-ACGCGGGCACGGCTGTCCAAGG-3' (forward) and 5'-GGCGCTCGCGGATGGCGCTGA-3' (reverse). ApoE will be amplified using the following conditions: 1 cycle of $98^{\circ} \mathrm{C}$ for $10 \mathrm{~s}, 35$ cycles of $72^{\circ} \mathrm{C}$ for $5 \mathrm{~s}, 1$ cycle of $72^{\circ} \mathrm{C}$ for $5 \mathrm{~min}$. PCR was performed in a final volume of $30 \mu \mathrm{l}$, containing $10 \mathrm{pmol}$ of forward and reverse primers and $50 \mathrm{ng}$ of genomic DNA template, using PrimeSTAR HS DNA Polymerase with GC Buffer (Takara Bio, Kusatsu, Shiga, Japan). 


\section{Plasma $\beta$-amyloid}

Recently, some studies found that plasma amyloid level was correlated with cognitive capacity and cerebrospinal fluid amyloid protein. ${ }^{445}$ In particular, when predicting brain amyloid deposition, the accuracy of the composite biomarker (amyloid- $\beta$ precursor protein 669-711/ $\mathrm{A} \beta 1-42$ and $\mathrm{A} \beta 1-40 / \mathrm{A} \beta 1-42$ ratios) could be approximately equal to $90 \%{ }^{46}$ Therefore, we would like to identify whether the plasma $\beta$-amyloid is also associated with cognitive decline in SCD individuals. Plasma A $\beta$ will be determined using a commercially available kit, V-PLEX A $\beta$ Peptide Panel 1 (6E10) Kit (K15200E) (Mesoscale Diagnostics, Rockville, Maryland, USA). A $\beta$ peptide levels from each blood draw will be measured in duplicate using the same aliquot.

\section{Urine tests}

Ten millilitres of first morning voided urine will be collected from each subject and then immediately refrigerated. An ELISA kit will be used to detect the protein level of AD7c-NTP in the urine specimens.

\section{Imaging protocol}

MRI data will be acquired using an integrated simultaneous 3.0 T TOF PET/MR (SIGNA PET/MR, GE Healthcare, Milwaukee, Wisconsin, USA) at the Xuanwu Hospital of Capital Medical University. For each participant, simultaneous PET and 3.0 T MRI data will be obtained. Brain MR images will be inspected by an experienced neuroradiologist.

PET: All participants will be invited for optional $\left[{ }^{18} \mathrm{~F}\right]$ florbetapir (AV-45) and $\left[{ }^{18} \mathrm{~F}\right]$ fluorodeoxyglucose (FDG) PET in 3-dimensional acquisition mode. The time duration between the FDG-PET and AV-45 PET is at least 3 days to eliminate the effect of the first tracer. For FDG-PET, each subject will be instructed to fast for at least 6 hours and must have a confirmed serum glucose level below $8 \mathrm{mmol} / \mathrm{L}$; $35 \mathrm{~min}$ dynamic scan is acquired approximately $40 \mathrm{~min}$ after an intravenous injection of $3.7 \mathrm{MBq} / \mathrm{kg}$ of $18 \mathrm{~F}-\mathrm{FDG}$. For A $\beta-\mathrm{PET}$, a $35 \mathrm{~min}$ dynamic scan is acquired approximately $40 \mathrm{~min}$ after an intravenous injection of $7-10 \mathrm{mCi}\left[{ }^{18} \mathrm{~F}\right]$ florbetapir. The PET data are acquired using a TOF-OSEM algorithm (timeof-flight ordered subset expectation maximisation) with the following parameters: eight iterations, 32 subsets matrix $=192 \times 192$, field of view $(\mathrm{FOV})=350 \times 350$, half-width height $=3$.

SMRI: Parameters for T1-weighted 3D brain structural images are as follows: $\mathrm{SPGR}$ sequence, $\mathrm{FOV}=256 \times 256 \mathrm{~mm}^{2}$, matrix $=256 \times 256$, slice thickness $=1 \mathrm{~mm}$, gap $=0$, slice number $=192$, repetition time $(\mathrm{TR})=6.9 \mathrm{~ms}$, echo time $(\mathrm{TE})=2.98 \mathrm{~ms}$, inversion time $(\mathrm{TI})=450 \mathrm{~ms}$, flip angle $=12^{\circ}$, voxel size $=1 \times 1 \times 1 \mathrm{~mm}^{3}$.

DTI: DTI data are obtained with a single-shot spin-echo diffusion-weighted echo planar imaging (EPI) sequence with the following parameters: FOV $=224 \times 224 \mathrm{~mm}^{2}$, data matrix $=112 \times 112$, slice thickness $=2 \mathrm{~mm}$, gap $=0$, slice number $=70$, slice order=interleaved, $\mathrm{TR}=16500 \mathrm{~ms}$,
$\mathrm{TE}=95.6 \mathrm{~ms}, 30$ gradient directions and $5 \mathrm{~b} 0$ images $\left(b=1000 \mathrm{~s} / \mathrm{mm}^{2}\right)$, voxel size $=2 \times 2 \times 2 \mathrm{~mm}^{3}$.

Rs-fMRI: A single-shot gradient-echo EPI sequence is used for rs-fMRI with the following parameters: scan duration $=8 \mathrm{~min}, \mathrm{FOV}=224 \times 224 \mathrm{~mm}^{2}$, data matrix $=64 \times 64$, slice thickness $=4.0 \mathrm{~mm}$, gap $=1.0 \mathrm{~mm}$, slice number $=28$, slice order=interleaved, TR=2000 $\mathrm{ms}, \quad \mathrm{TE}=30 \mathrm{~ms}$, flip angle $=90^{\circ}$, voxel size $=3.5 \times 3.5 \times 4 \mathrm{~mm}^{3}$.

T-fMRI: All participants will also be invited for a block design fMRI paradigm to measure somatosensory mismatch negativity (MMN). We will use a custom-designed air jet pressure stimulation device, which can apply 5.0 bar pressure somatosensory stimulation to the right index or middle finger. The index and middle finger of the right hand will be fixed in place with two pieces of adhesive tapes to restrict any movement during the experiment. The length of the two air pipes from the air compressor to the apertures will be $6.0 \mathrm{~m}$, and the diameter of the two stimulation apertures will be 1.0 $\mathrm{mm}$. In addition, the distance between the aperture and the centre of the finger pad will be fixed at $5.0 \mathrm{~mm}$ for all subjects. Subjects will be instructed to watch a video (without sound) presented on a custom designed screen and to ignore stimulation to the fingers.

The functional MRI paradigm will have a total duration of $9 \mathrm{~min}$. In a block design, eight control blocks with $32 \mathrm{~s}$ duration and $8 \mathrm{MMN}$ blocks (also 32s duration) will be used. A constant stimulus onset asynchrony of $500 \mathrm{~ms}$ will be employed for both the MMN and control conditions resulting in 40 stimuli per block. In devising the MMN and control blocks, we will take advantage of an MMN paradigm in which two stimuli will be presented equiprobably and their presentation order will be varied. In MMN blocks, stimulation will be arranged to compose alternating 'mini-sequences' of stimulus 1 (index finger, $5.0 \mathrm{bar}, 300 \mathrm{~ms}$ ) and 2 (middle finger, $5.0 \mathrm{bar}, 300 \mathrm{~ms}$ ). Mini-sequences will be 2, 3, or 4 repetitions of a single stimulus (each sequence length is represented equiprobably), followed by a mini-sequence of the other stimuli, and so on. The number of trials in a given mini-sequence will vary such that the occurrence of a switch from stimulus 1 to stimulus 2 (and vice-versa) is irregular. As such, the switch trial stimuli will be designated as deviants and will elicit the MMN. This basic stimulation paradigm, which has proven to elicit robust MMNs, will be used for the MMN condition. ${ }^{47} 48$ For the matched control condition to the aforementioned MMN condition, the same two stimuli will be alternated sequentially to form a regular pattern (eg, stimulus 1, stimulus 2, stimulus 1, stimulus 2, etc.).

\section{Imaging data analysis}

PET data analysis. The AV-45 and FDG-PET scans will be preprocessed by Statistical Parametric Mapping V.12 (SPM 12; https://www.fil.ion.ucl.ac.uk/spm/software/ spm12). The structural images will be individually registered to the averaged PET images. The unified segmentation method ${ }^{49}$ will then be applied to all coregistered 
structural images. The PET images will be spatially normalised to Montreal Neurological Institute (MNI) standard space by using the forward parameters estimated during the unified segmentation and smoothed with an $8 \mathrm{~mm}$ full width at half maximum (FWHM) Gaussian kernel. Finally, the voxel-wise AV-45 and FDG-PET standardised uptake value ratio (SUVR) will be normalised by the whole cerebellum and pons as the reference regions, respectively. A global AV-45 PET SUVR value will be estimated using a composite of the prefrontal, orbitofrontal, parietal, temporal, anterior cingulate, and posterior cingulate and precuneus cortices.

SMRI data analysis. The cortical thickness analysis will be performed using the FreeSurfer image analysis suite, V.5.3 (http://surfer.nmr.mgh.harvard.edu). We will construct models of the boundaries between the grey matter (GM) and the white matter as well as the pial surface. ${ }^{50}$ Cortical thickness measures will be obtained by calculating the distance between these surfaces. Automated reconstruction and labelling will be performed using the default 'recon-all' command line. All generated images will be visually inspected for image and segmentation quality and corrected manually if necessary. Subsequently, 'lobesStrict' will be performed to obtain a lobar annotation and consecutively imported for further statistical analyses. For the voxel-based morphometry analysis, structural images will be segmented into GM tissue using the new segment function within SPM12. The diffeomorphic anatomical registration through the exponentiated Lie algebra toolbox will be used to generate a reference template object of the sample that will be warped into a standard MNI space. The generated flow fields of each subject will be calculated and normalisation parameters will be then implemented to normalise the GM maps in the native space to the MNI space. During the normalisation process, the modulated images (local native amount of GM) will be preserved. Images will be spatially smoothed with an $8 \mathrm{~mm}$ FWHM Gaussian kernel. Finally, for each individual, we will obtain a smoothed GM volumetric map. Additionally, the total intracranial volume for each individual will be estimated by summing the segmented GM, white matter and cerebral spinal fluid (CSF). Then, the smoothed GM images of every individual will be used for subsequent statistical analysis.

DTI data analysis. Raw DTI data will be processed using the Oxford Centre for Functional MRI of the Brain (FMRIB) Software Library (FSL, http://www.fmrib.ox.ac. $\mathrm{uk} / \mathrm{fsl}) \cdot{ }^{51-53}$ Initially, the EddyCorrect tool will be used to correct eddy current distortions and motion artefacts by fine registration of the DTI images to a reference image (b0 image). The Brain Extraction Tool ${ }^{53}$ will be used for creating brain masks of all subjects, and then a diffusion tensor will be modelled at each voxel by using the leastsquares algorithm fitting tensor model within the DTI-FIT Tool. ${ }^{54}$ Fractional anisotropy (FA), mean diffusivity (MD), radial diffusivity $(\mathrm{RD})$ and axial diffusivity $(\mathrm{AxD})$ values of each voxel will be calculated based on the eigenvalues of the tensor. Voxel-wise statistical analysis of the FA, MD,
$\mathrm{RD}$ and $\mathrm{AxD}$ data will be performed using tract-based spatial statistics. ${ }^{55}$ All subjects' FA maps will be non-linearly coregistered to the FMRIB58_FA template with FSL's non-linear image registration algorithm. Then, the mean FA image will be obtained and thinned to create a mean FA skeleton representing the centre of all tracts common to all subjects. Each subject's aligned FA data will then be projected onto the FA skeleton to obtain their FA skeletons and deformation matrixes. With the deformation matrixes, the skeletonized $\mathrm{AxD}, \mathrm{MD}$ and $\mathrm{RD}$ maps will be created for every individual by the tbss_non_FA tool. These maps will be used for subsequent statistical analysis.

Rs-fMRI data analysis. Rs-fMRI will be preprocessed using the Data Processing Assistant for Resting-state fMRI (DPASF; http://www.rfmri. org/DPARSF) ${ }^{56}$ The first 10 volumes will be discarded for image stabilisation and the participant's to adaptation to the scanning. The remaining functional sequences will be first corrected for timing differences between each slice and motion effects. Next, the structural image will be coregistered to the mean functional image. Then, the transformed structural images will be segmented into GM, white matter and CSF. The motion-corrected functional volumes will be spatially normalised to MNI 152 standard space and resampled to $3 \mathrm{~mm} \times 3 \mathrm{~mm} \times 3 \mathrm{~mm}$ cubic voxels by using the normalisation parameters estimated during unified segmentation. The resulting images will further undergo spatial smoothing (Gaussian kernel with $8 \mathrm{~mm}$ FWHM), linear detrending and temporal filtering $(0.01-0.08 \mathrm{~Hz})$. To avoid overestimating regional homogeneity (ReHo) values, spatial smoothing will be conducted for individual ReHo maps rather than during data preprocessing. Finally, nuisance signals (including Friston 24-head motion parameters, the white matter and CSF) will be extracted and regressed out from the data to reduce the residual effects of non-neuronal factors. For the amplitude of low frequency fluctuation (ALFF) analysis, ${ }^{57}$ the time series of each voxel will be transformed into the frequency domain using a fast Fourier transform. The square root of the power spectrum will be calculated and averaged across $0.01-0.08 \mathrm{~Hz}$. This averaged square root will be taken as the ALFF value for this voxel. For the ReHo analysis, ${ }^{58}$ Kendall's coefficient of concordance (KCC) will be computed on the ranked time series of a given voxel with its 26 nearest neighbours. The resultant KCC will be taken as the ReHo values. The generated ALFF and ReHo images will be used for statistical analysis.

Network analysis. We will construct a structural cortical network based on GM volumes. ${ }^{59}$ The nodes will be defined as brain regions corresponding to automated anatomic labelling (AAL) areas. The structural connections will be defined as statistical correlations between pairs of average GM volumes in our study. A structural connection will be considered to exist if the correlation coefficient is statistically significant. Before the correlation analysis, the effects of age, sex and total GM volume on the GM volume of regions will be adjusted. We will calculate Pearson correlation coefficients across 
individuals between the average GM of every pair of regions, and then an interregional correlation matrix will be obtained for every individual. We will construct a white matter network based on fibre number. The network nodes will be defined as the 90 regions of interest corresponding to the AAL template. The weight of the network edge will be defined as the number of connected fibres with two regions. The fibre number will be calculated through DTI tractography as reported in our previous study. ${ }^{60}$ To avoid spurious connections, a minimum threshold of fibre number (weight of the edge $=10$ ) will be used. We will construct a functional network based on the average time sequence. The network nodes will be defined as the 90 regions of interest corresponding to the AAL template. The network edge will be defined as the partial correlation coefficients between the average time sequence of two regions, and we will obtain an incidence matrix. Non-significant correlations will be excluded. The network analyses will be performed with the GRETNA toolbox (http://www.nitrc.org/projects/gretna/). ${ }^{61}$ The 'rich-club coefficient' is defined as the density of connections between rich-club nodes and rich-club regions in our study will be defined as the top 13 brain regions with the highest degree as reported in our previous study. ${ }^{62}$ A module is defined as a subset of nodes connected to the other nodes in the same module other than those outside the module. ${ }^{63}$ We plan to use Newman's metric to measure the modularity ${ }^{64}$ and maximise the modularity parameter $Q$ by the algorithm proposed by Clauset et $a l^{65}$ The two parameters will be calculated for the structural cortical network, white matter network and functional network constructed for each individual. The values of the two measures will be calculated for every individual and used in the model construction.

T-fMRI data analysis. We will use SPM12 to process and analyse the t-fMRI data. The first 10 volumes will be discarded due to unsteady magnetization. First, the functional images from each run will be realigned. The structural image will then be coregistered to the first scan in the functional image, and the resulting coregistered structural image will be normalised to MNI 152 standard space. Finally, these spatially normalised functional images will be smoothed (Gaussian kernel with an $8 \mathrm{~mm}$ FWHM).

\section{Statistical analysis}

Baseline comparison between SCD converters and SCD nonconverters

All data (demographic information, clinical data, risk factors, neuropsychologic tests, blood and urine biomarkers, multimodal MRI biomarkers, glucose mentalism and amyloid deposition) will be described. The Shapiro-Wilk test and Q-Q plots will be used to confirm the normality. All normally distributed continuous variables will be reported as the mean $\pm \mathrm{SD} A$ comparison of the baseline between SCD converters and SCD non-converters will be performed with a two-sample t-test for continuous variables and a chi-squared test for categorical variables. Dunnett's multiple comparison tests will be performed for comparison.

Statistical analyses of t-fMRI data will be subjected to a general linear model analysis. The MMN condition will serve as the predictor of interest and will be modelled with a boxcar function convolved with the canonical haemodynamic response function; the Control condition will serve as a baseline. The beta estimates from the individual general linear models enter a second level random effects analysis. Contrast maps will be created by applying paired t-tests comparing the MMN versus control condition for each group separately as well as a two-sample t-test for the between-groups comparison. Activation maps will be corrected $(\mathrm{p}<0.001)$ by the false discovery rate approach implemented in SPM.

\section{Longitudinal patterns in SCD converters}

The longitudinal analysis in neuropsychological, plasma, urine and MRI variables in SCD-converters will be assessed. We will use the general linear mixed effects model to estimate the individual's change in each variable.

\section{Statistical prediction models}

We will use the competing risk regression model to detect the association between possible risk factors and endpoint. The endpoint event of our study is converting to MCI. Death before conversion to MCI is considered a competing risk in our study. The time to event is the date difference between the baseline neuropsychological tests and the date at which the MCI or dementia is first diagnosed.

Independent variables are listed in table 2, including features of baseline clinical characteristics, blood, urine, MRI and PET biomarkers. Considering the overfitting effect when establishing the model, the least absolute shrinkage and selection operator (LASSO) model with penalty parameter tuning will be used for variable screening. It is a data mining method for shrinkage estimation and dimensionality reduction, overcoming processing difficulties caused by high-dimensional data, and estimating the parameters more accurately. Then, we will perform the sub-distribution hazard function model, which could evaluate hazards for the endpoint (MCI or dementia) and competing (death) events, to establish the final multivariate models. Ten-fold cross-validation is used to perform internal verification of the established models. The optimism-corrected C-statistics will be used to evaluate the performance of risk prediction models.

To better clarify the relationship between possible risk factors and endpoint events, some confounding factors will be adjusted. Four models will be established in our study. Model 1 will simply take demographic data, lifestyle, clinical assessment, neuropsychological assessments and SCD report into account. Model 2 will add the ApoE genotype, plasma $\mathrm{A} \beta$ and urine $\mathrm{AD} 7 \mathrm{c}-\mathrm{NTP}$ on the basis of model 1. Model 3 will take neuroimaging data into account on the basis of model 2 and model 4 will include amyloid-PET and FDG-PET on the basis of model 3. 


\begin{tabular}{|c|c|}
\hline Types & \\
\hline Demographic data & $\begin{array}{l}\text { Age; education level; family history of } \\
\text { dementia; socioeconomic information }\end{array}$ \\
\hline Lifestyle & $\begin{array}{l}\text { Nicotine and alcohol use; sleep quality } \\
\text { (PSQI, RBDSQ, ESS); nutrition style }\end{array}$ \\
\hline Clinical assessment & $\begin{array}{l}\text { Vascular risk factors (hypertension, } \\
\text { hyperlipemia, diabetes, coronary } \\
\text { heart disease, Fazekas score); medial } \\
\text { temporal lobe atrophy scale; HAMA; } \\
\text { HAMD; GDS }\end{array}$ \\
\hline $\begin{array}{l}\text { Neuropsychological } \\
\text { assessment }\end{array}$ & $\begin{array}{l}\text { AVLT-D; AVLT-R; STT-A; STT-B; BNT; } \\
\text { AFT }\end{array}$ \\
\hline SCD report & $\begin{array}{l}\text { Onset time; comparison with others; } \\
\text { SCD-Q9; ECog; consistency of SCD; } \\
\text { inform report }\end{array}$ \\
\hline Blood tests & ApoE genotype; Plasma $\beta$-amyloid \\
\hline Urine tests & Level of AD7c-NTP \\
\hline MRI data & $\begin{array}{l}\text { Cortical thickness; grey matter volume; } \\
\text { FA; MD; RD; AxD; ALFF; ReHo; } \\
\text { network characteristic (rich-club } \\
\text { coefficient, modality); MMN-related } \\
\text { haemodynamic responses }\end{array}$ \\
\hline PET data & Global SUVR of FDG-PET and A $\beta$-PET \\
\hline
\end{tabular}

AFT, animal fluency test; ALFF, amplitude of low-frequency fluctuations; AVLT, Auditory Verbal Learning Test; AxD, axial diffusivity; $A \beta-P E T, \beta$-amyloid positron emission tomography; BNT, Boston naming test; ECog, Everyday Cognition; ESS, Epworth Sleepiness Scale; FA, fractional anisotropy; FDG-PET, fluorodeoxyglucose positron emission tomography; GDS, Geriatric Depression Scale; HAMA, the Hamilton Anxiety Scale; HAMD, the Hamilton Depression Scale; MD, mean diffusivity; MMN, mismatch negativity; PET, positron emission tomography; PSQI, Pittsburgh sleep quality index; RBDSQ, Rapid Eye Movement Sleep Behaviour Disorder Screening Questionnaire; RD, radial diffusivity; ReHo, regional homogeneity; SCD, subjective cognitive decline; STT, Shape Trails Test; SUVR, standardised uptake volume ratios.

The HRs with $95 \%$ confidence intervals are determined. A two-sided $p<0.05$ is defined as statistical significance. All statistical tests were performed using R statistical software. The 'glmnet' package will be used for the LASSO model analysis, and the 'cmprsk' package will be performed for the sub-distribution hazard function model.

\section{DISCUSSION}

The current study evaluates the characteristics of SCD patients presenting cognitive decline within 5 years. Furthermore, we will construct risk forecast models based on different combinations of potential predictors to achieve early diagnosis in the preclinical stage of AD.

Considering the long preclinical stage of $\mathrm{AD}$ and compensated cognitive function in the SCD stage, the application of a refined SCD approach as an enrichment strategy for clinical trials focusing on preclinical AD shows great promise. SILCODE is a longitudinal study, and we will focus on features of SCD converters with multi-perspective analysis. In particular, we would like to establish an integrated diagnostic system for the early detection and prediction of SCD progressing at the individual level. We aim to provide scientific evidence for a more effective diagnosis of $\mathrm{SCD}$ due to $\mathrm{AD}$ and explore the underlying mechanism.

In this project, plasma $\mathrm{A} \beta$, urine $\mathrm{AD} 7 \mathrm{c}-\mathrm{NTP}$, and multimodal MRI will also be included in the predicting models. Plasma and urine biomarkers are non-invasive and feasible in clinical practice. Previous studies have found their diagnostic efficiency in MCI and dementia, ${ }^{46}{ }^{66}$ but in SCD individuals, there are few pieces of evidence. Therefore, this project also focuses on new biomarkers and tries to identify their usage in the clinical scene. Additionally, through this project, we would like to reveal the longitudinal patterns of involved clinical and MRI biomarkers, especially the temporal sequences, which may provide a basis for us to understand the changes during disease progression.

The stage model theory proposes that human memory can be divided into three stages: sensory memory, shortterm memory, and long-term memory. ${ }^{67}$ The MMN has been proposed as an objective measure of the existence of auditory (visual, somatosensory) sensory memory traces. ${ }^{68}$ Previous studies have suggested that MMN may have the potential to measure the age-related changes ${ }^{47}$ or improve the diagnostic value for the early diagnosis of $\mathrm{AD} .{ }^{69}$ Although many more SCD studies have concentrated on short-term memory and long-term memory, ${ }^{18} 70$ there are very few published studies on the sensory memory stage. SILCODE will investigate the changes in the initial memory stage in the SCD population and the predictive value of somatosensory MMN for disease progression in an exploratory way.

Finally, cultural factors were found to impact SCD by a cross-cultural comparison between the USA and China, ${ }^{21}$ which indicates the need for SCD research in China. SILCODE is a longitudinal study in China, and the implementation of SILCODE would provide characteristics of SCD in China, which contribute to the harmonisation of the SCD concept across cultural borders.

\section{Author affiliations}

${ }^{1}$ Department of Neurology, Xuanwu Hospital of Capital Medical University, Beijing, China

${ }^{2}$ Department of Psychiatry, University of Cambridge, Cambridge, UK

${ }^{3}$ Sino-Britain Centre for Cognition and Ageing Research, Faculty of Psychology, Southwest University, Chonging, China

${ }^{4}$ Department of Psychiatry and Psychotherapy, Medical Faculty, University of Cologne, Cologne, Germany

${ }^{5}$ Center of Alzheimer's Disease, Beijing Institute for Brain Disorders, Beijing, China

${ }^{6}$ Beijing Institute of Geriatrics, Beijing, China

${ }^{7}$ National Clinical Research Center for Geriatric Disorders, Beijing, China

Acknowledgements The authors would like to thank Kun Yang for helping them to revise the statistical section of the manuscript. The authors would like to thank the participants of this study and patient advisers for their support.

Contributors $\mathrm{XL}$ and $\mathrm{XW}$ are joint first authors. $\mathrm{XL}$ and $\mathrm{XW}$ are responsible for the implementation of the trial and manuscript draft and revision. LS is responsible for 
the design and implementation of imaging data analysis. $\mathrm{XH}$ is responsible for the design of SCD and clinical assessments. YH is responsible for the study design, implementation of the trial, manuscript draft and revision, and funding. All authors contributed to the drafting of the manuscript and approved the final manuscript.

Funding This work was supported by The National Key Research and Development Program of China (2016YFC1306300), National Natural Science Foundation of China (Grant 61633018, 81430037), Beijing Nature Science Foundation (7161009), Beijing Municipal Commission of Health and Family Planning (PXM2019_026283_000002), German Research Council (DFG; Grant number: JE 2707/7-1), The Sino-German Center for Research Promotion (Grant Number: GZ1370, GZ1425). Li Su is supported by Alzheimer's Research UK (ARUK-SRF2017B-1).

\section{Competing interests None declared.}

Patient consent for publication Not required.

Ethics approval Ethical approval of this has been obtained from the medical research ethics committee and institutional review board of the Xuanwu Hospital, Capital Medical University (ID: [2017]046).

Provenance and peer review Not commissioned; externally peer reviewed.

Open access This is an open access article distributed in accordance with the Creative Commons Attribution Non Commercial (CC BY-NC 4.0) license, which permits others to distribute, remix, adapt, build upon this work non-commercially, and license their derivative works on different terms, provided the original work is properly cited, appropriate credit is given, any changes made indicated, and the use is non-commercial. See: http://creativecommons.org/licenses/by-nc/4.0/.

\section{REFERENCES}

1. Scheltens P, Blennow K, Breteler MM, et al. Alzheimer's disease. Lancet 2016;388:505-17.

2. Jack CR, Knopman DS, Jagust WJ, et al. Hypothetical model of dynamic biomarkers of the Alzheimer's pathological cascade. Lancet Neurol 2010:9:119-28.

3. Honig LS, Vellas B, Woodward M, et al. Trial of solanezumab for mild dementia due to Alzheimer's disease. N Engl J Med 2018;378:321-30.

4. Doody RS, Thomas RG, Farlow M, et al. Phase 3 trials of solanezumab for mild-to-moderate Alzheimer's disease. $N$ Engl J Med 2014;370:311-21.

5. Dubois B, Hampel H, Feldman $\mathrm{HH}$, et al. Preclinical Alzheimer's disease: definition, natural history, and diagnostic criteria. Alzheimers Dement 2016;12:292-323.

6. Jessen F, Amariglio RE, van Boxtel M, et al. A conceptual framework for research on subjective cognitive decline in preclinical Alzheimer's disease. Alzheimers Dement 2014;10:844-52.

7. Molinuevo JL, Rabin LA, Amariglio R, et al. Implementation of subjective cognitive decline criteria in research studies. Alzheimers Dement 2017;13:296-311.

8. Rabin LA, Smart CM, Amariglio RE. Subjective cognitive decline in preclinical Alzheimer's disease. Annu Rev Clin Psychol 2017;13:369-96.

9. Mitchell AJ, Beaumont H, Ferguson D, et al. Risk of dementia and mild cognitive impairment in older people with subjective memory complaints: meta-analysis. Acta Psychiatr Scand 2014;130:439-51

10. Dardenne S, Delrieu J, Sourdet S, et al. Memory complaints and cognitive decline: data from the GUIDAGE study1. J Alzheimers Dis 2017;60:1567-78.

11. Jessen $\mathrm{F}$, Wolfsgruber $\mathrm{S}$, Wiese $\mathrm{B}$, et al. AD dementia risk in late $\mathrm{MCl}$, in early $\mathrm{MCl}$, and in subjective memory impairment. Alzheimers Dement 2014:10:76-83.

12. Buckley RF, Maruff P, Ames D, et al. Subjective memory decline predicts greater rates of clinical progression in preclinical Alzheimer's disease. Alzheimers Dement 2016;12:796-804.

13. Hessen $E$, Eckerström M, Nordlund A, et al. Subjective cognitive impairment is a predominantly benign condition in memory clinic patients followed for 6 years: the gothenburg-oslo $\mathrm{MCl}$ study. Dement Geriatr Cogn Dis Extra 2017;7:1-14.

14. Vogel JW, Varga Doležalová M, La Joie R, et al. Subjective cognitive decline and $\beta$-amyloid burden predict cognitive change in healthy elderly. Neurology 2017;89:2002-9.

15. Bessi V, Mazzeo S, Padiglioni S, et al. From subjective cognitive decline to Alzheimer's disease: the predictive role of neuropsychological assessment, personality traits, and cognitive reserve. A 7-year follow-up study. J Alzheimers Dis 2018;63:1523-35.

16. Fernández-Blázquez MA, Ávila-Villanueva M, Maestú F, et al. Specific features of subjective cognitive decline predict faster conversion to mild cognitive impairment. J Alzheimers Dis 2016;52:271-81.
17. Wolfsgruber S, Kleineidam L, Wagner M, et al. Differential risk of incident Alzheimer's disease dementia in stable versus unstable patterns of subjective cognitive decline. J Alzheimers Dis 2016;54:1135-46.

18. Jessen F, Spottke A, Boecker H, et al. Design and first baseline data of the DZNE multicenter observational study on predementia Alzheimer's disease (DELCODE). Alzheimers Res Ther 2018;10:15.

19. Slot RER, Verfaillie SCJ, Overbeek JM, et al. Subjective cognitive impairment cohort (SCIENCe): study design and first results. Alzheimers Res Ther 2018;10:76.

20. Rodriguez-Gomez O, Sanabria A, Perez-Cordon A, et al. FACEHBI: a prospective study of risk factors, biomarkers and cognition in a cohort of individuals with subjective cognitive decline. study rationale and research protocols. J Prev Alzheimers Dis 2017;4:100-8.

21. Wu Q. Subjective cognitive impairment of older adults: a comparison between the US and China. Int J Methods Psychiatr Res 2016;25:68-75.

22. Jackson JD, Rentz DM, Aghjayan SL, et al. Subjective cognitive concerns are associated with objective memory performance in Caucasian but not African-American persons. Age Ageing 2017;46:988-93.

23. Hao L, Wang X, Zhang L, et al. Prevalence, risk factors, and complaints screening tool exploration of subjective cognitive decline in a large cohort of the Chinese population. $J$ Alzheimers Dis 2017;60:371-88.

24. Rabin LA, Smart CM, Crane PK, et al. Subjective cognitive decline in older adults: an overview of self-report measures used across 19 international research studies. J Alzheimers Dis 2015;48(Supp 1):S63-86.

25. Perquin M, Diederich N, Pastore J, et al. Prevalence of dementia and cognitive complaints in the context of high cognitive reserve: a population-based study. PLoS One 2015;10:e0138818.

26. Gifford KA, Liu D, Romano R, et al. Development of a subjective cognitive decline questionnaire using item response theory: a pilot study. Alzheimers Dement 2015;1:429-39.

27. Tomaszewski Farias S, Mungas D, Harvey DJ, et al. The measurement of everyday cognition: development and validation of a short form of the Everyday Cognition scales. Alzheimers Dement 2011;7:593-601.

28. Chen $\mathrm{KL}, \mathrm{Xu} \mathrm{Y}, \mathrm{Chu} \mathrm{AQ}$, et al. Validation of the Chinese version of montreal cognitive assessment basic for screening mild cognitive impairment. J Am Geriatr Soc 2016;64:e285-e90.

29. Hsieh FY, Lavori PW. Sample-size calculations for the cox proportional hazards regression model with nonbinary covariates. Control Clin Trials 2000;21:552-60.

30. Licher S, Leening MJG, Yilmaz P, et al. Development and validation of a dementia risk prediction model in the general population: an analysis of three longitudinal studies. Am J Psychiatry 2019;176.

31. Pinto TCC, Machado L, Bulgacov TM, et al. Influence of age and education on the performance of elderly in the brazilian version of the montreal cognitive assessment battery. Dement Geriatr Cogn Disord 2018;45(5-6):290-9.

32. Bondi MW, Edmonds EC, Jak AJ, et al. Neuropsychological criteria for mild cognitive impairment improves diagnostic precision, biomarker associations, and progression rates. J Alzheimers Dis 2014;42:275-89.

33. Zhao Q, Lv Y, Zhou Y, et al. Short-term delayed recall of auditory verbal learning test is equivalent to long-term delayed recall for identifying amnestic mild cognitive impairment. PLoS One 2012;7:e51157.

34. Guo Q, Hong Z, Shi W, et al. Boston naming test in Chinese elderly, patient with mild cognitive impairment and Alzheimer's dementia. Chinese Mental Health Journal 2006;20:81-4.

35. Guo Q, Jin L, Hong Z, et al. A specific phenomenon of animal fluency test in Chinese elderly. Chinese Mental Health Journal 2007;21:622-5

36. Zhao Q, Guo Q, Li F, et al. The Shape Trail Test: application of a new variant of the Trail making test. PLoS One 2013;8:e57333.

37. McKhann GM, Knopman DS, Chertkow H, et al. The diagnosis of dementia due to Alzheimer's disease: recommendations from the National Institute on Aging-Alzheimer's Association workgroups on diagnostic guidelines for Alzheimer's disease. Alzheimers Dement 2011;7:263-9.

38. Bhome R, Huntley JD, Price G, et al. Clinical presentation and neuropsychological profiles of Functional Cognitive Disorder patients with and without co-morbid depression. Cogn Neuropsychiatry 2019;24.

39. Buckley R, Saling M, Ellis K, et al. Self and informant memory concerns align in healthy memory complainers and in early stages of mild cognitive impairment but separate with increasing cognitive impairment. Age Ageing 2015;44:1012-9. 
40. Buysse DJ, Reynolds CF, Monk TH, et al. The pittsburgh sleep quality index: a new instrument for psychiatric practice and research. Psychiatry Res 1989;28:193-213.

41. Nomura T, Inoue Y, Kagimura T, et al. Utility of the REM sleep behavior disorder screening questionnaire (RBDSQ) in Parkinson's disease patients. Sleep Med 2011;12:711-3.

42. Johns MW. A new method for measuring daytime sleepiness: the Epworth sleepiness scale. Sleep 1991;14:540-5.

43. Guo QH, Zhou B, Zhao QH, et al. Memory and Executive Screening (MES): a brief cognitive test for detecting mild cognitive impairment. BMC Neurol 2012;12:119.

44. Llado-Saz S, Atienza M, Cantero JL. Increased levels of plasma amyloid-beta are related to cortical thinning and cognitive decline in cognitively normal elderly subjects. Neurobiol Aging 2015;36:2791-7.

45. Hanon O, Vidal JS, Lehmann S, et al. Plasma amyloid levels within the Alzheimer's process and correlations with central biomarkers. Alzheimers Dement 2018;14:858-68.

46. Nakamura A, Kaneko N, Villemagne VL, et al. High performance plasma amyloid- $\beta$ biomarkers for Alzheimer's disease. Nature 2018;554:249-54.

47. Strömmer JM, Tarkka IM, Astikainen P. Somatosensory mismatch response in young and elderly adults. Front Aging Neurosci 2014;6:293

48. Shinozaki N, Yabe H, Sutoh T, et al. Somatosensory automatic responses to deviant stimuli. Brain Res Cogn Brain Res 1998;7:165-71.

49. Ashburner J, Friston KJ. Unified segmentation. Neuroimage 2005;26:839-51.

50. Fischl B, van der Kouwe A, Destrieux C, et al. Automatically parcellating the human cerebral cortex. Cereb Cortex 2004;14:11-22.

51. Smith SM, Jenkinson M, Woolrich MW, et al. Advances in functional and structural MR image analysis and implementation as FSL. Neuroimage 2004;23(Suppl 1):S208-19.

52. Woolrich MW, Jbabdi S, Patenaude B, et al. Bayesian analysis of neuroimaging data in FSL. Neuroimage 2009;45(1 Suppl):S173-86.

53. Smith SM. Fast robust automated brain extraction. Hum Brain Mapp 2002:17:143-55.

54. Basser PJ, Mattiello J, LeBihan D. MR diffusion tensor spectroscopy and imaging. Biophys $J$ 1994;66:259-67.

55. Smith SM, Jenkinson M, Johansen-Berg H, et al. Tract-based spatial statistics: voxelwise analysis of multi-subject diffusion data. Neuroimage 2006;31:1487-505

56. Chao-Gan Y, Yu-Feng Z. DPARSF: a MATLAB Toolbox for "Pipeline" data analysis of resting-state fMRI. Front Syst Neurosci 2010;4:13.
57. Zang YF, He Y, Zhu CZ, et al. Altered baseline brain activity in children with ADHD revealed by resting-state functional MRI. Brain Dev 2007;29:83-91.

58. Zang Y, Jiang T, Lu Y, et al. Regional homogeneity approach to fMRI data analysis. Neuroimage 2004;22:394-400.

59. Yao Z, Zhang Y, Lin L, et al. Abnormal cortical networks in mild cognitive impairment and Alzheimer's disease. PLoS Comput Biol 2010;6:e1001006.

60. Shu N, Wang X, Bi Q, et al. Disrupted topologic efficiency of white matter structural connectome in individuals with subjective cognitive decline. Radiology 2018;286:229-38.

61. Wang J, Wang X, Xia M, et al. GRETNA: a graph theoretical network analysis toolbox for imaging connectomics. Front Hum Neurosci 2015;9:386

62. Yan T, Wang W, Yang L, et al. Rich club disturbances of the human connectome from subjective cognitive decline to Alzheimer's disease. Theranostics 2018;8:3237-55.

63. Radicchi F, Castellano C, Cecconi F, et al. Defining and identifying communities in networks. Proc Natl Acad Sci U S A 2004;101:2658-63.

64. Newman ME. Fast algorithm for detecting community structure in networks. Phys Rev E Stat Nonlin Soft Matter Phys 2004;69(6 Pt 2):066133.

65. Clauset A, Newman ME, Moore C. Finding community structure in very large networks. Phys Rev E Stat Nonlin Soft Matter Phys 2004;70(6 Pt 2):066111.

66. Zhang J, Zhang CH, Li RJ, et al. Accuracy of urinary AD7c-NTP for diagnosing Alzheimer's disease: a systematic review and metaanalysis. J Alzheimers Dis 2014;40:153-9.

67. McGaugh JL. Time-dependent processes in memory storage. Science 1966;153:1351-8

68. Näätänen R. Mismatch negativity: clinical research and possible applications. Int J Psychophysiol 2003;48:179-88.

69. Papaliagkas VT, Anogianakis G, Tsolaki MN, et al. Progression of mild cognitive impairment to Alzheimer's disease: improved diagnostic value of the combined use of N200 latency and beta-amyloid(1-42) levels. Dement Geriatr Cogn Disord 2009;28:30-5.

70. Erk S, Spottke A, Meisen A, et al. Evidence of neuronal compensation during episodic memory in subjective memory impairment. Arch Gen Psychiatry 2011;68:845-52.

71. Rodda JE, Dannhauser TM, Cutinha DJ, et al. Subjective cognitive impairment: increased prefrontal cortex activation compared to controls during an encoding task. Int J Geriatr Psychiatry 2009;24:865-74. 